\title{
Paper
}

\section{The Portuguese Version of the Eating Disorders Inventory: Evaluation of its Psychometric Properties}

\author{
Paulo P. P. Machado*, Sonia Gonçalves, Carla Martins and \\ Isabel C. Soares \\ Department of Psychology, University of Minho, Braga, Portugal
}

The objective of the present study was to examine the psychometric properties of the Portuguese version of the Eating Disorders Inventory (EDI; Garner et al., 1983). Three groups participated in the current study: 274 female and 67 male non-patients, and 83 females with a diagnosis of eating disorder. Results clearly support the reliability and the factor structure of the EDI in a Portuguese sample. In addition non-patient and eating disordered participants' score significantly differently in all EDI scales, emphasizing the clinical usefulness of the measure. Copyright (C) 2001 John Wiley $\mathcal{E}$ Sons, Ltd and Eating Disorders Association.

\section{INTRODUCTION}

As in other countries, eating disorders have received increased clinical and research attention over recent years. Epidemiological studies carried out in Portugal (e.g. Azevedo and Ferreira, 1992; Carmo et al., 1996; Baptista et al., 1996) have suggested a lower prevalence of eating disorders when compared to that of other western countries. However, more recent studies with female college students (S. Gonçalves et al., presentation at the 30th Annual Meeting of the Society for Psychotherapy Research, Braga, Portugal, 1999) have found higher prevalence rates than previous studies.

One of the most widely used self-report instruments designed to measure psychological and behaviour traits associated with eating disorders is the Eating Disorders Inventory (EDI, Garner et al., 1983). The original EDI scale consisted of 64 items divided into eight subscales. Three of the scales assessed

\footnotetext{
* Correspondence to: Paulo P. P. Machado, Universidade do Minho, Departamento de Psicologia, Campus de Gualtar, P-4710 Braga, Portugal. 
attitudes and behaviours toward weight, eating, and body shape (Drive for Thinness, Bulimia, and Body Dissatisfaction). The other five subscales were designed to measure general psychological features of persons with eating disorders. These scales are Ineffectiveness, Perfection, Interpersonal Distrust, Interoceptive Awareness, and Maturity Fears.

The EDI has been translated into several languages including Portuguese, and has been used for clinical purposes, more recently in the context of the European collaborative study on eating disorders (Effective Treatment of Eating Disorders, COST Action b6; see Kordy and Treasure, 1998). Although several previous studies have established the reliability and validity of the EDI (see, Garner (1990) for a review) there was no study that established them for the Portuguese translation.

The purpose of the current study was to examine the psychometric characteristics of the Portuguese translation of the EDI and its usefulness for research and clinical use.

\section{METHOD}

\section{Subjects}

A total of 424 subjects participated in the current study. These subjects were drawn from both clinical and non-clinical populations. Two groups represented the non-clinical population. One group of high school students $(n=94)$ with ages ranging from 15 to 18 years $(M=16.05$ years, $S D=1.03$ years) and another of college students $(n=247)$ with ages ranging from 18 to 62 years $(M=21.94$ years, $\mathrm{SD}=5.20$ years $)$. Sixty-seven participants were males (19.6 per cent) and 274 females (80.4 per cent).

In addition, 55 patients with a diagnosis of anorexia nervosa, and 28 with a diagnosis of bulimia nervosa, who sought treatment in a specialized eating disorders unit participated in this study. Patients were diagnosed by one of the unit's psychiatrists according to DSM IV (APA, 1995) criteria. All patients were female, and ages ranged from 14 to 35 years $(M=16.59$ years, $\mathrm{SD}=3.87$ years).

Table 1 presents the characteristics of the three group samples that participated in the current study.

\section{Instruments}

\section{Eating Disorders Inventory (EDI; Garner et al., 1983)}

The EDI described above is a frequently used 64-item self-report measure of eating related attitudes and behaviours. Subjects are asked to respond to a 6point forced-choice format by rating how much the item applied to them. Options ranged from 'always' to 'never'. The most extreme eating disorder response earns a score of 3 , the intermediate response scores 2 , and the next response scores 1 ; the other three responses receive no score. Scale scores are 
Table 1. Demographic characteristics of the non-clinical and clinical population samples 
the sum of all items for that particular subscale. The Portuguese version of the EDI used in the current study was translated, for research purposes, into Portuguese by the clinical team of Santa Maria Hospital, in Lisbon. The original version of the EDI was translated into Portuguese by a bilingual clinical psychologist and then translated back into English by an independent professional.

\section{Data analysis strategy}

Internal consistency was evaluated by computing Cronbach's alphas for each of the eight subscales and the total score. A principal component factorial analysis with oblique rotation was performed on the non-clinical population data. The method used non-transformed item scores (i.e. ranging from 1 to 6, see Schoemaker et al., 1994), and data was forced into an 8-factor solution. Itemtotal correlations were computed for each of the 64 items. Finally, EDI results for each of the clinical and non-clinical samples were compared, and a cut-off point computed.

\section{RESULTS}

\section{Reliability}

Cronbach's alpha, item subscale, and item-total scale correlations are presented in Table 2. Alpha coefficients were 0.93 for the total scale, and ranged from 0.69 to 0.91 for the subscales: 0.91 (Drive for Thinness), 0.81 (Interpersonal Distrust), 0.69 (Perfectionism), 0.81 (Bulimia), 0.73 (Maturity Fears), 0.81 (Interoceptive Awareness), 0.91 (Body Dissatisfaction) and 0.88 (Ineffectiveness). Table 2 presents the item-subscale, and item-total score correlations as well as the Cronbach alpha for all the subscales. Using a cut-off reliability coefficient of 0.70 , as indicated by the alpha coefficients of all the subscales except Perfectionism, appears to have adequate reliability.

\section{Factorial analysis}

The resulting eight factors, reproduced those obtained by Garner et al. (1983), and explained 47 per cent of the total variance. Only two items $(35,47)$ did not load onto any factor. Item 35 ('The demands of adulthood are too great') had a loading of -0.2594 on factor 5 (original subscale of the item); and, item 47 ('I feel bloated after eating a small meal') had a loading inferior to 0.30 . Table 2 shows the factor loadings for an 8-factor solution.

\section{Correlational analysis}

Table 3 presents the correlation matrix between the subscales of the EDI. 
Table 2. Factor analysis of the Eating Disorders Inventory (EDI) in a nonclinical population $(N=341)$

Loadings

Factor 1 (Drive for Thinness)

1. I eat sweets and carbohydrates without feeling nervous*

$-0.34949$

7. I think about dieting

0.71529

11. I feel extremely guilty after overeating

0.65049

16. I am terrified of gaining weight

0.72942

25. I exaggerate the importance of weight

0.56986

32. I am preoccupied with the desire to be thinner

0.73507

49. If I gain a pound, I worry that I will keep gaining

0.69452

64. When I am upset, I worry that I will start eating

0.59156

Factor 2 (Interpersonal Distrust)

15. I am open about my feelings*

0.70057

17. I trust others*

0.47432

23. I can communicate with others easily*

0.48261

30. I have close relationships*

0.43233

34. I have trouble expressing my emotions to others

$-0.62889$

54. I need to keep people at a certain distance

$-0.63833$

57. I can talk about personal thoughts or feelings*

0.73032

Factor 3 (Perfectionism)

13. Only outstanding performance is good enough in my family

0.40068

29. As a child, I tried very hard to avoid disappointing my parents

0.62154 and teachers

36. I hate being less than best at things

0.31411

43. My parents have expected excellence of me

0.61261

52. I feel that I must do things perfectly or not do them at all

0.45529

63. I have extremely high goals

0.47224

Factor 4 (Bulimia)

4. I eat when I am upset

$-0.31226$

5. I stuff myself with food

$-0.67953$

28. I have gone on eating binges where I have felt that I could not stop

38. I think about bingeing

$-0.58180$

46. I eat moderately in front of others and stuff myself when they are gone

53. I have the thought of trying to vomit in order to lose weight

$-0.85201$

61. I eat or drink in secrecy

$-0.36413$

Factor 5 (Maturity Fears)

3. I wish that I could return to the security of childhood

$-0.51451$

6. I wish that I could be younger

$-0.50760$

14. The happiest time in life is when you are a child

$-0.42532$

$-0.46048$

22. I would rather be an adult than a child*

0.58925

39. I feel happy that I am not a child anymore*

0.63325

48. I feel that people are happiest when they are children

$-0.50148$

58. The best years of your life are when you become an adult* 0.53908

Factor 6 (Interoceptive Awareness)

8. I get frightened when my feelings are too strong

0.47123

21. I get confused about what emotion I am feeling

0.75794

26. I can clearly identify what emotion I am feeling*

$-0.46407$

33. I don't know what's going on inside me

0.56014 


\section{Table 2. Continued}

\begin{tabular}{lr}
\hline & Loadings \\
\hline 40. I get confused as to whether or not I am hungry & 0.32601 \\
44. I worry that my feelings will get out of control & 0.46153 \\
51. When I am upset, I don't know if I am sad, frightened, or angry & 0.58852 \\
60. I have feelings I can't quite identify & 0.54247 \\
Factor 7 (Body Dissatisfaction) & \\
2. I think that my stomach is too big & 0.39835 \\
9. I think that my things are too large & 0.50736 \\
12. I think that my stomach is just the right size* & -0.51855 \\
19. I feel satisfied with the shape of my body* & -0.59448 \\
31. I like the shape of my buttocks* & -0.70614 \\
45. I think my hips are too big & 0.53445 \\
55. I think that my thighs are just the right size* & -0.74807 \\
59. I think my buttocks are too large & 0.63550 \\
62. I think that my hips are just the right size* & -0.70917 \\
Factor 8 (Ineffectiveness) & \\
10. I feel ineffective as a person & 0.60573 \\
18. I feel alone in the world & 0.42978 \\
20. I feel generally in control of things in my life* & -0.42253 \\
24. I wish I were someone else & 0.54796 \\
27. I feel inadequate & 0.55936 \\
37. I feel secure about myself* & -0.61837 \\
41. I have a low opinion of myself & 0.51647 \\
42. I feel that I can achieve my standards* & -0.46919 \\
50. I feel that I am a worthwhile person* & -0.62941 \\
56. I feel empty inside (emotionally) & 0.31349 \\
\hline
\end{tabular}

Items 35 (The demands of adulthood are too great) and 47 (I feel bloated after eating a small meal) are not scored within any factor. *Scored in reverse direction.

Some subscales were significantly intercorrelated. All eight subscales correlated with the EDI total scores (Pearson's correlation ranged from 0.445 for Perfectionism to 0.810 for Drive for Thinness, $p<0.0001)$. Item-total correlations ranged from 0.29 for item 1 to 0.84 for item $16(p<0.0001)$.

\section{Discriminant validity}

The aim of discriminant validity is to determine to what degree a clinical population with eating disorders (anorexia nervosa and bulimia nervosa) differs on each subscale of the EDI from a group of normal control subjects.

Table 4 presents the total and subscale score means and standard deviations for each subject group. For comparison purposes only the female subgroup of the non-clinical sample was included in the analysis, because the eating disorder subgroup included only female participants.

A multivariate analysis of variance (MANOVA) on the EDI scales revealed an overall effect of the group $(F(8,343)=136.46, p<0.01)$. Subsequent subscale 
Table 3. Correlation matrix between subscales

\begin{tabular}{lrrrccccc}
\hline & DT & ID & P & B & MF & IA & BD & I \\
\hline DT & \multicolumn{1}{c}{1.00} & & & & & & & \\
ID & -0.0701 & 1.00 & & & & & & \\
P & 0.0038 & -0.0358 & 1.00 & & & & & \\
B & -0.2406 & 0.1132 & -0.1876 & 1.00 & & & & \\
MF & -0.0697 & 0.1629 & -0.0978 & 0.1513 & 1.00 & & & \\
IA & 0.4479 & -0.0554 & 0.0937 & -0.2693 & -0.1024 & 1.00 & & \\
BD & 0.2840 & -0.4285 & -0.0360 & -0.1535 & -0.1560 & 0.2537 & 1.00 & \\
I & 0.2097 & -0.3251 & 0.2233 & -0.3311 & -0.2364 & 0.1306 & 0.3141 & 1.00 \\
\hline
\end{tabular}

DT, Drive for Thinness; ID, Interpersonal Distrust; P, Perfectionism; B, Bulimia; MF, Maturity Fears; IA, Interoceptive Awareness; BD, Body Dissatisfaction; I, Ineffectiveness.

by subscale univariate analysis of variance (ANOVA) revealed significant differences between groups on all subscales, respectively: Drive for Thinness $(F(2,350)=82.56, p<0.01)$; Bulimia $(F(2,350)=140.86, p<0.01)$; Body Dissatisfaction $(F(2,350)=21.72, p<0.01)$; Ineffectiveness $(F(2,350)=92.39$, $p<0.01)$; Perfectionism $(F(2,350)=11.60, p<0.01)$; Interpersonal Distrust $(F(2,350)=27.77, p<0.01)$; Interoceptive Awareness $(F(2,350)=66.57, p<0.01)$; and, Maturity Fears $(F(2,350)=22.09, p<0.01)$.

Post-hoc Scheffé tests revealed that all groups scored significantly differently $(p<0.01)$ on the Drive for Thinness, Bulimia, Body Dissatisfaction, and Ineffectiveness subscales. Bulimics scored highest on all these subscales, and anorectics scored higher than non-clinical subjects. For the remaining subscales the two clinical groups scored higher than their non-clinical counterparts but not significantly differently from each other.

\section{Cut-off score}

Further analysis was performed to determine a cut-off score that allowed discrimination between a clinical and non-clinical population. To determine this score we used the formula provided by Jacobson and Truax (1991) for clinically significant change. This formula ${ }^{1}$ provides a cut-off point that represents the point where a subject is closer to the mean of the normal sample than to the mean of the clinical population, and vice versa.

Considering the mean and the standard deviation of our clinical sample (anorexic and bulimic) and of the non-clinical sample we calculated the cut-off score between the clinical and non-clinical group. The computed cut-off score was 43 for the total score of the EDI.

\footnotetext{
${ }^{1} \mathcal{C}=\mathrm{S} 0 \mathrm{M} 1+\mathrm{S} 1 \mathrm{M} 0 / \mathrm{S} 0+\mathrm{S} 1$ where $\mathrm{M} 0=$ mean of the normal population, $M 1=$ mean of the clinical group, and S0, S1 = standard deviations of the normal and clinical group.
} 
Table 4. Mean scores and SD for subscales and total score by subgroup

\begin{tabular}{|c|c|c|c|c|c|}
\hline & \multicolumn{3}{|c|}{ Nonclinical population } & \multirow[b]{2}{*}{$\begin{array}{c}\text { Anorexia } \\
(N=55)\end{array}$} & \multirow[b]{2}{*}{$\begin{array}{c}\text { Bulimia } \\
(N=28)\end{array}$} \\
\hline & $\begin{array}{l}\text { Total sample } \\
\qquad(N=341)\end{array}$ & $\begin{array}{c}\text { Men } \\
(N=67)\end{array}$ & $\begin{array}{c}\text { Women } \\
(N=274)\end{array}$ & & \\
\hline Drive for Thinness & $3.19(4.71)$ & $0.81(1.39)$ & $3.77(5.04)$ & $8.78(6.91)$ & $15.21(4.70)$ \\
\hline Bulimia & $1.14(2.22)$ & $0.82(1.94)$ & $1.26(2.37)$ & $2.75(4.53)$ & $11.55(6.06)$ \\
\hline Body Dissatisfaction & $5.65(6.27)$ & $2.40(3.06)$ & $6.45(6.59)$ & $9.11(5.65)$ & $14.77(8.53)$ \\
\hline Ineffectiveness & $1.61(2.71)$ & $0.94(1.69)$ & $1.78(2.88)$ & $8.22(7.24)$ & $11.44(9.07)$ \\
\hline Perfectionism & $4.56(3.57)$ & $5.59(3.01)$ & $4.31(3.65)$ & $6.00(3.80)$ & $7.52(5.00)$ \\
\hline Interpersonal Distrust & $2.90(3.11)$ & $2.67(2.68)$ & $2.96(3.21)$ & $5.85(3.99)$ & $6.81(5.41)$ \\
\hline Interoceptive Awareness & $3.08(4.05)$ & $3.28(3.91)$ & $3.04(4.09)$ & $8.53(6.00)$ & $10.37(7.92)$ \\
\hline Maturity Fears & $4.82(3.67)$ & $5.09(4.51)$ & $4.76(3.44)$ & $8.51(5.83)$ & $7.55(6.49)$ \\
\hline Total & $26.85(18.59)$ & 21.57 (11.69) & $28.10(19.69)$ & $59.24(28.71)$ & $88.88(36.07)$ \\
\hline
\end{tabular}




\section{DISCUSSION}

The internal consistency estimates for seven of the eight subscales of the EDI were in the acceptable range with Cronbach alpha ranging from 0.69 for the Perfectionism subscales up to 0.91 for the Drive for Thinness and Body Dissatisfaction subscales.

The 8-factor solution replicated that obtained by Garner et al. (1983). Only two of the 64 items did not load primarily on their original scale.

The scale also differentiated the non-clinical and clinical subgroups. And, four of the scales (Drive for Thinness, Bulimia, Body Dissatisfaction, and Ineffectiveness) differentiated not only the clinical and non-clinical subgroups, but also the bulimic and anorectic subgroups. Bulimic patients scored higher than anorectic patients and also higher than non-clinical female participants. In addition anorectic patients scored higher than non-clinical participants.

In summary, the results of the current study support the reliability, validity and clinical usefulness of the Portuguese version of The Eating Disorder Inventory.

\section{ACKNOWLEDGEMENTS}

This study was partially supported by a grant of Fundação para a Ciência e a Technologia/Foundation for Science and Technology, Portugal (PRAXIS/ $\mathrm{PCSH} / \mathrm{P} / \mathrm{PSI} / 85 / 96$ ) to the first author.

We wish to express our appreciation to Professor Daniel Sampaio, Professor Isabel do Carmo and the clinical team of the Eating Disorder Unit of the Santa Maria Hospital in Lisbon (Portugal) for sharing their clinical data.

\section{REFERENCES}

American Psychiatric Association (APA). 1995. Diagnostic and Statistical Manual of Mental Disorders (4th edn). American Psychiatric Association: Washington, DC.

Azevedo MH, Ferreira CP. 1992. Anorexia nervosa and bulimia: a prevalence study. Acta Psychiatrica Scandianavia 86: 432-436.

Baptista F, Sampaio D, Carmo I, Reis D, Galvão-Teles A. 1996. The prevalence of disturbance of eating behaviour in a Portuguese female university population. European Eating Disorders Review 4: 260-270.

Carmo I, Reis D, Varandas P, Bouça D, Padre Santo D, Neves A, André I, Sampaio D, Galvão-Teles A. 1996. Prevalence of anorexia nervosa: a portuguese population study. European Eating Disorders Review 4: 157170.

Garner DM. 1990. Eating Disorder Inventory - 2: Professional Manual. WPS: Los Angeles. 
Garner DM, Olmsted MP, Polivy J. 1983. Development and validation of a multidimensional Eating Disorder Inventory for anorexia nervosa and bulimia. International Journal of Eating Disorder 2: 15-34.

Jacobson NS, Truax P. 1991. Clinical significance: a statistical approach to defining meaningful change in psychotherapy research. Journal of Consulting and Clinical Psychology 59: 12-19.

Kordy H, Treasure J. 1998. Effectiveness and efficiency of psychotherapy treatment programs: The European collaborative longitudinal observational study on eating disorders (ECLOS-ED). In Psychotherapeutic Issues on Eating Disorders: Models, Methods and Results, Bria P, Ciocca A (eds). Abramowicz: Roma, Italy; 1-18.

Schoemaker C, Strien T, Staak C. 1994. Validation of the Eating Disorder Inventory in a nonclinical population using transformed and untransformed responses. International Journal of Eating Disorders 15: 387-393. 